\title{
ALDO ROSSI EN SEVILLA: LOS PRIMEROS VIAJES (1975-1978)
}

\author{
Victoriano Sainz Gutiérrez \\ Aldo Rossi in Seville: the first journeys (1975-1978) \\ Boletín Académico. Revista de investigación y arquitectura contemporánea \\ Escuela Técnica Superior de Arquitectura. Universidade da Coruña \\ elSSN 2173-6723 \\ unw.boletinacademico.com \\ Número 3 (2013) \\ Páginas 01-14 \\ Fecha de recepción 01.10.2012 \\ Fecha de aceptación 02.02.2013
}

https://doi.org/10.17979/bac.2013.3.0.991

\section{Resumen}

El presente artículo quiere establecer la cronología y las circunstancias de los primeros viajes a Sevilla de Aldo Rossi en la segunda mitad de la década de 1970. En esas estancias sevillanas del arquitecto milanés se originó un doble influjo: de una parte, se di-fundieron entre los arquitectos locales las ideas rossianas sobre la arquitectura y la ciudad; de otra, el propio Rossi pudo profundizar en la cultura y la arquitectura andaluzas. Ese conocimiento no tardaría en convertir a Sevilla en lugar de memoria y en fuente de 'objetos de afecto' para sus personalísimas arquitecturas.

\section{Abstract}

This paper attempt to establish the chronology and circumstances of the Aldo Rossi's first journeys to Seville in the second half of the 1970s. In this Rossi's Sevillian stays arose a double influence: on the one hand, Rossi's ideas about architecture and the city were diffused among the local architects; on the other, even Rossi himself could get inside the Andalusian culture and architecture. This knowledge soon converts Seville into a place of memory and source of 'objects of affection' for his very personal works.

\section{Palabras clave}

Aldo Rossi, Sevilla, Espańa, Viajes de arquitectos, Influencia italiana

\section{Keywords}

Aldo Rossi, Seville, Spain, Architects' journeys, Italian influence 

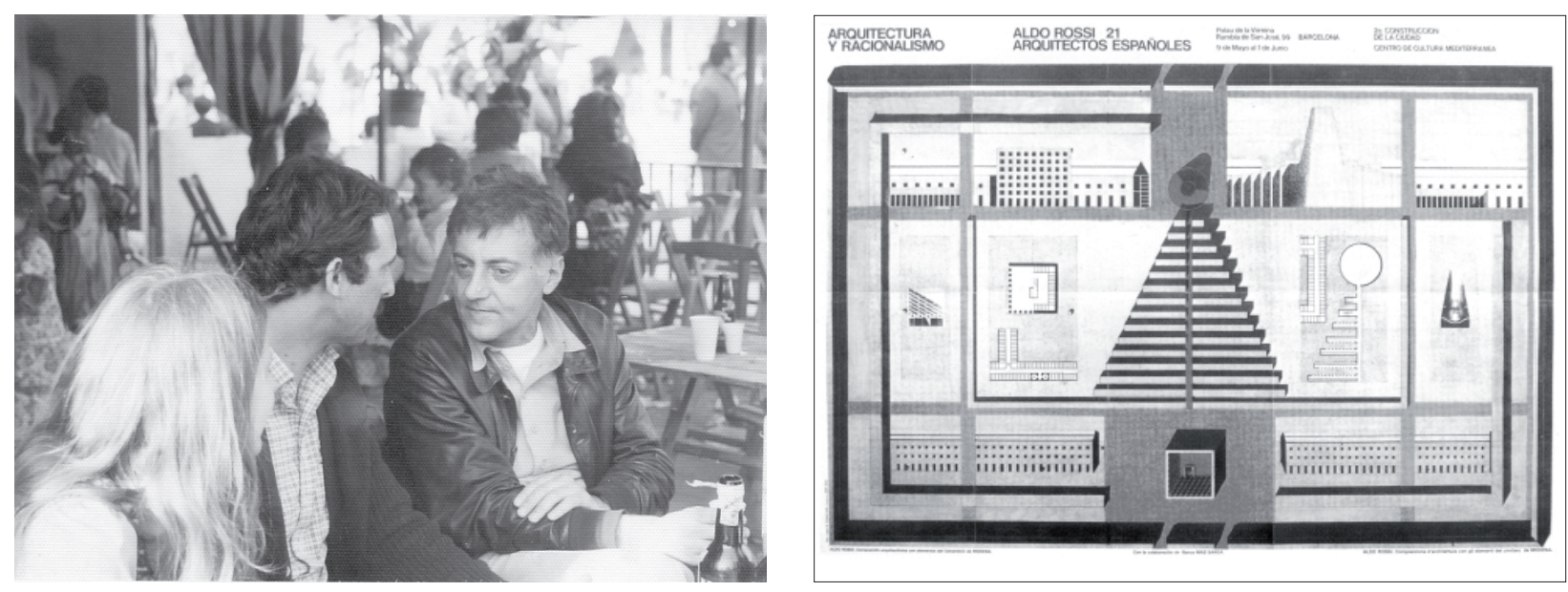

1 Aldo Rossi y Guillermo Vázquez Consuegra en la Feria de Sevilla (1975)

2 Cartel de la exposición "Aldo Rossi + 21 arquitectos españoles", Barcelona (1975)

La relación de Aldo Rossi con España se remonta a mediados de la década de 1960. En septiembre de 1964, durante un viaje a Barcelona acompańado por Guido Canella, tuvo ocasión de conocer la capital catalana y establecer contacto con Salvador Tarragó Cid. En los ańos siguientes Rossi pudo visitar diversas regiones españolas, lo cual le permitió conocer de primera mano la arquitectura de nuestras ciudades, llegando a convertirse, según él mismo solía decir ${ }^{1}$, en un auténtico hispanista: «Empiezo a conocer España casi como Italia y tengo ganas de conocerla cada vez mejor», escribía al arquitecto catalán en 1970, después de un viaje por el norte de la península ibérica² ${ }^{2}$.

Los contactos de Rossi con arquitectos españoles se intensificaron de manera especial a lo largo de los años setenta, en gran parte gracias a la amplia actividad de difusión de sus ideas llevada a cabo por Tarragó. La estrecha amistad surgida entre ambos, alimentada por el deseo que Rossi siempre tuvo de no quedar encerrado en los limitados confines del contexto italiano, contribuyó a que el maestro lombardo adquiriera una temprana notoriedad en España y a que sus planteamientos sobre la arquitectura y la ciudad calaran hondo en nuestra cultura disciplinar.

Tres fueron las vías a través de las cuales se produjo esa penetración del pensamiento rossiano en nuestro país: la traducción de su libro La arquitectura de la ciudad, editado por Gustavo Gili en 197133 la creación de la revista $2 C$ Construcción de la Ciudad, cuyo primer número apareció en $1972^{4}$; y las conferencias impartidas por Rossi entre 1972 y 1976 en diversas ciudades españolas, por invitación de los respectivos Colegios de Arquitectos 5 . En los tres casos el impulso de Tarragó resultó decisivo: él fue quien se encargó de traducir el libro y de gestionar su publicación; él fue asimismo quien fundó el Grupo 2C, promotor de la revista homónima, y quien organizó unos encuentros periódicos de las comisiones de cultura de los Colegios de Arquitectos, que sirvieron también para dar a conocer la figura de Rossi ${ }^{6}$.

\section{SEVILLA, «PATRIA DE ELECCIÓN» PARA ROSSI}

Además de Cataluña, tres fueron los ámbitos geográficos en los que el influjo de Rossi se dejó sentir con especial fuerza: Galicia, donde la figura del arquitecto pontevedrés César Portela Fernández-Jardón resultó determinante; el País Vasco, a través principalmente de los arquitectos donostiarras José Ignacio Linazasoro Rodríguez y Miguel Garay Ormazábal; y Andalucía, una región llamada a jugar un papel del todo singular en la obra rossiana, como lo ha reconocido un sector de la crítica cuando, al analizar su arquitectura, ha senáado que las formas empleadas por el maestro lombardo, "por su carácter, son fundamentalmente noritalianas con un componente andaluz añadido, que refleja el especial amor de Rossi por el sur de España» ${ }^{7}$.

Los primeros viajes de Rossi a Andalucía datan de esos mismos años sesenta. La Mezquita de Córdoba y la Alhambra de Granada le impresionaron particularmente, porque vio en ellas una confirmación de la teoría de la ciudad que entonces estaba desarrollando; de hecho, de esas arquitecturas monumentales se ocupa en el primer 
capítulo «La arquitectura de la ciudad», al analizar la estructura de los hechos urbanos ${ }^{8}$. En el verano de 1965, tras haber visitado ambas ciudades y todavía bajo el impacto de esa experiencia, escribe al amigo catalán: «La experiencia humana que he tenido en Andalucía me ha conmovido y me incita cada vez más a conocer y estudiar todos los aspectos de vuestro gran país»?

Pero serían las sucesivas visitas a Sevilla en la segunda mitad de los años setenta, donde creó una amplia red de relaciones con arquitectos locales, las que le permitieran profundizar en ese conocimiento de la cultura andaluza; un conocimiento que pronto convirtió a esta ciudad en fuente de imágenes y de claves de lectura para su personalísima obra. Y es que Sevilla estaba llamada a impresionarle de una manera muy particular, hasta el punto de llegar a «convertirse para él en patria de elección» ${ }^{10}$. El arquitecto milanés encontró en la capital andaluza una cultura popular, atravesada por un sentido a la vez festivo y trágico de la vida, que le fascinaría; de ahí que no dudara en afirmar que la Feria y la Semana Santa eran "las mayores arquitecturas que jamás he conocido» ${ }^{11}$.

Con su capacidad para descubrir modos de relacionar cosas aparentemente dispares entre sí, en sus paseos por la ciudad Rossi no tardó en percibir similitudes entre la arquitectura milanesa y la sevillana: «Las relaciones analógicas, las asociaciones entre cosas y situaciones, no han hecho sino aumentar en mis sucesivas estancias en Andalucía, tal como en este momento, mezclando autobiografía e historia civil, emergen en mí las imágenes de la estructura de la casa sevillana. Siempre me ha gustado la tipología del corral y la he representado muchas veces: el corral era la forma de vida de las casas del viejo Milán» ${ }^{12}$.

Las analogías no tardarían en multiplicarse. Todo en la ciudad le hablaba de un mundo que le resultaba muy familiar: si los corrales de vecinos le evocaron inmediatamente las casas di ringhiera milanesas, algo semejante le sucedió con las imágenes de la Semana Santa, que le trajeron a la memoria las figuras de los sacri monti de su infancia, o con las palmeras, que no podían dejar de recordarle las de un hotel de Mergozzo, junto al lago Mayor, donde a menudo se retiraba para descansar. Pero esas relaciones analógicas funcionaban también en sentido inverso, y así las cabinas de las playas de Elba pronto quedarían asociadas a las casetas de la Feria sevillana. De este modo se fue configurando un imaginario que pronto entraría de lleno en su arquitectura, ayudándole a dar forma a algunos de sus proyectos más característicos de esos años.
Los viajes de Rossi a Sevilla se concentraron en dos momentos claramente diferenciados: la segunda mitad de los años setenta, coincidiendo con el desarrollo de su proyecto para el Corral del Conde, y la segunda mitad de los años ochenta, en que recibiría dos nuevos encargos profesionales en la capital andaluza ${ }^{13}$. De entre los muchos arquitectos sevillanos con los que entabló relación en esos años, hay dos que tuvieron una importancia particular: Guillermo Vázquez Consuegra, que le invitó por primera vez a la ciudad y mantuvo con el maestro lombardo un estrecho contacto sobre todo en los primeros viajes, y Fernando Villanueva Sandino, quien con su desinteresada amistad le proporcionaría los tres proyectos que Rossi redactó para la ciudad del Guadalquivir ${ }^{14}$.

Tanto para la arquitectura sevillana como para el arquitecto milanés fueron fundamentales los acontecimientos vividos en Sevilla en la segunda mitad de los años setenta: unos años especialmente relevantes para la afirmación internacional de la figura de Rossi, que comenzó por entonces a viajar a los Estados Unidos, pero también un momento crucial en el despegue profesional de una generación de arquitectos locales, algunos de los cuales andando el tiempo se convertirían en figuras destacadas de la arquitectura española. Ciertamente, Rossi siguió visitando Sevilla en la segunda mitad los años ochenta, pero para entonces las circunstancias eran otras, en la vida de Rossi y en la de la ciudad; los años decisivos habían transcurrido ya.

Esta vinculación de Rossi con Sevilla no ha sido sistemáticamente investigada hasta la fecha, quizá porque la ausencia de fuentes disponibles constituye una notable limitación para ello. No es de extrañar, por tanto, que los primeros intentos de explorarla contengan no pocas imprecisiones y errores, sobre todo en lo referente a las circunstancias y las fechas en que se produjo el desembarco del arquitecto milanés en la capital andaluza. A intentar establecerlas con el rigor y la fiabilidad que son posibles en este momento está dedicado este artículo, que pretende ofrecer una base cierta desde la que aproximarse al significado de esas estancias sevillanas de Rossi.

\section{5: DOS CONFERENCIAS Y UN PROYECTO}

A comienzos de los años setenta un activo grupo de jóvenes arquitectos sevillanos había comenzado a tomar el relevo de la generación anterior, formada por profesionales con oficio y buen hacer, pero cuya carrera había tocado fondo. La presencia creciente de estos 
jóvenes en el Colegio de Arquitectos, como miembros de sus organismos de gestión, y en la Escuela de Arquitectura, como profesores de casi todas las áreas de conocimiento, les iba a permitir asumir un notable protagonismo en la vida cultural de la ciudad, a través de la contestación a unos modos de hacer que habían producido un evidente deterioro urbanístico, y de la propuesta de enfoques alternativos abiertos a los nuevos aires que llegaban desde el exterior. En este sentido, sería determinante para el establecimiento de nuevas relaciones el hecho de que, junto a una mayoría de egresados de la Escuela de Sevilla, algunos hubieran acabado la carrera en Madrid o Barcelona ${ }^{15}$.

Hay que destacar a este respecto la labor desarrollada desde la comisión de cultura del Colegio de Arquitectos, que a partir de 1973 mantuvo un estrecho contacto con las comisiones de otros Colegios, estableciéndose así una dinámica común que cristalizaría más adelante en los Seminarios Internacionales de Arquitectura Contemporánea (SIAC). En el caso sevillano, esa comisión había empezado a organizar actividades desde su constitución en 1970, pero fue en 1974 cuando adquirió una estructura más articulada, que le permitiría diversificar sus competencias y multiplicar las actividades. El Centro de Estudios y Servicios (CEYS), creado entonces, sirvió para que los arquitectos pudieran intervenir en el debate ciudadano a través de una serie de conferencias, mesas redondas, exposiciones, estudios monográficos y pronunciamientos, que significaron la apertura de un cauce para la participación pública en un contexto político todavía no democrático ${ }^{16}$.

Dentro de este marco se inscribe la venida de Rossi a Sevilla en abril de 1975. Invitado por el CEYS para dar unas conferencias, habló como es lógico de su arquitectura, pero también de los problemas de los centros históricos, uno de los temas estrella del momento. De hecho, la defensa del patrimonio había sido la cuestión desencadenante de esas reuniones de las comisiones de cultura de los Colegios de Arquitectos, impulsadas por Tarragó. En la primera de ellas, celebrada en Palma de Mallorca en mayo de 1972, se había elaborado un documento, conocido como «Declaración de Palma», que marcó la pauta para el trabajo de los años sucesivos; allí se animaba a los Colegios de Arquitectos a organizarse e implicarse activamente en la tarea, dando directrices muy concretas para llevar a cabo ese trabajo ${ }^{17}$. A la tercera de esas reuniones, que tuvo lugar en Santiago de Compostela en diciembre de 1973, acudió Rossi para dar una conferencia en la que expuso su particular modo de entender la inter- vención en la ciudad histórica; entre los asistentes se encontraban Juan Ruesga Navarro y Fernando Villanueva, como responsables del recién creado archivo histórico del Colegio de Arquitectos sevillano ${ }^{18}$.

Rossi no era, pues, un desconocido en Sevilla. Si se le invitó a dar aquellas conferencias es porque algunos miembros del CEYS estaban familiarizados con sus ideas, que eran para ellos una clara referencia en su tarea docente en la Escuela: Antonio Barrionuevo Ferrer, Francisco Torres Martínez o Guillermo Vázquez Consuegra se hallaban estudiando las tipologías del centro histórico con sus alumnos de Elementos de Composición, mientras que Villanueva y Ruesga venían utilizando para sus clases de Urbanismo los planteamientos recogidos en el libro colectivo sobre la ciudad de Padua ${ }^{19}$. Todo ello explica que, al no encontrar hotel para alojar a Rossi, Vázquez Consuegra pidiera a Villanueva que lo hospedase en su casa; a partir de entonces, cada vez que visitó la capital andaluza hizo por alojarse en ese chalé del barrio de Heliópolis ${ }^{20}$. Comenzó ahí una entrañable amistad entre ambos, que pronto daría paso a una colaboración profesional sólo interrumpida en 1992 con la prematura muerte del arquitecto sevillano.

La primera estancia de Rossi en Sevilla forma parte de un viaje más amplio por la península ibérica, del que conocemos las fechas de comienzo y final porque Rossi las dejó consignadas en uno de sus cuadernos azules: «13 de abril-15 de mayo, España y Portugal ${ }^{21}$. Rossi llegaba a Sevilla el sábado 19 de abril, en plena Feria (Fig. 01), para dar dos conferencias que fueron concebidas como un ciclo inicialmente denominado "Teoría de la arquitectura y crítica ideológica ${ }^{22}$. La primera de esas conferencias, titulada «Algunos de mis proyectos», era una presentación de sus obras en el contexto de la arquitectura análoga que se encontraba desarrollando por entonces; la segunda, dedicada a "Cuestiones de los centros históricos», retomaba algunos temas ya expuestos en su intervención de 1973 en Santiago. Como el Colegio de Arquitectos no andaba sobrado de recursos, la demarcación de Sevilla acordó compartir los gastos del viaje con las de Granada y Córdoba, a cambio de que el arquitecto milanés diera sendas conferencias a sus respectivos colegiados ${ }^{23}$.

Esa estancia permitió a Rossi no sólo conocer la arquitectura de la capital andaluza, que le impresionó profundamente por las correspondencias existentes entre algunas tipologías residenciales de Sevilla y Milán, sino también apreciar determinadas manifestaciones 

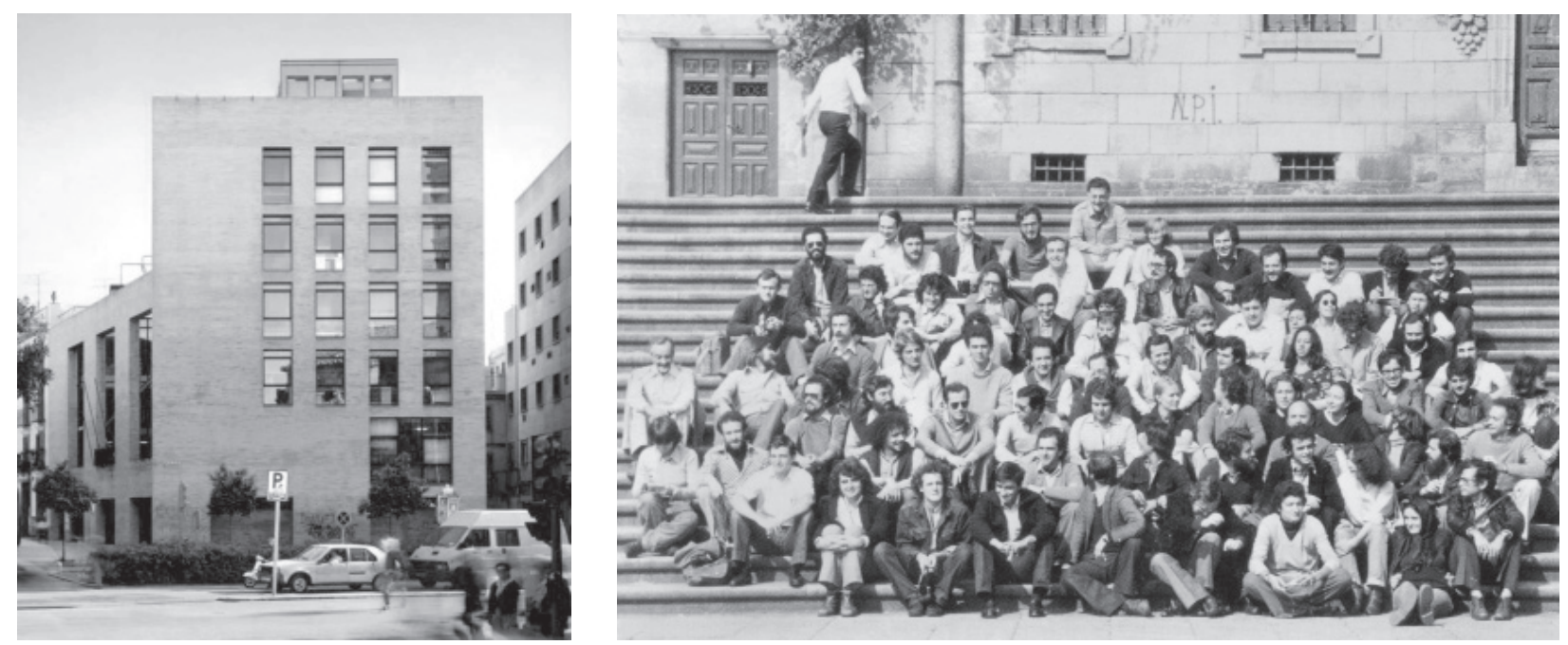

3 Gabriel Ruiz Cabrero y Enrique Perea, Sede del Colegio Oficial de Arquitectos, Sevilla (1976-79) 4 Aldo Rossi con los asistentes al I SIAC, Santiago de Compostela (1976)

de la cultura popular, como la Feria o la Semana Santa, que dejaron honda huella en su arquitectura, porque en cierta medida le eran congeniales. Así lo explicaba él mismo en el hermosísimo texto escrito como introducción para un libro que los responsables del CEYS pensaron publicar con motivo de aquel viaje: la rigidez de unos elementos que se repiten, absolutamente previsibles, constituye el marco que posibilita el desarrollo de todo lo que de imprevisible hay en la vida; ésta, que es una constante en el discurso arquitectónico de Rossi, resulta ser también la lección que vio confirmada en Sevilla ${ }^{24}$.

Pero el primer viaje a Sevilla había de depararle todavía otra sorpresa: el encargo de un proyecto. El Corral del Conde, luego declarado monumento nacional, se encontraba en un estado ruinoso y había sido recientemente adquirido por Pro-Sevilla, la peculiar inmobiliaria promovida por el duque de Segorbe con el fin salvar de la piqueta una parte del caserío de la ciudad histórica ${ }^{25}$. Pro-Sevilla había ido comprando numerosos inmuebles en el sector de Santiago y había encargado a OTAISA (Oficinas Técnicas de Arquitectura e Ingeniería S.A.) la redacción de un plan especial que definiera una estrategia de intervención para el conjunto del barrio. Conocedores del entusiasmo que habían despertado en el arquitecto milanés los corrales de vecinos sevillanos, Fernando Villanueva y Gonzalo Díaz Recasens, que entonces trabajaban en OTAISA, sugirieron al administrador de Pro-Sevilla, Ignacio Medina, la posibilidad de que se le encargase la rehabilitación del histórico corral; Rossi aceptó encantado ${ }^{26}$.

\section{6: EL JURADO DE UN CONCURSO}

En abril de 1976 Rossi realizó un nuevo viaje a Sevilla. La ocasión que lo hizo posible fue la convocatoria de un concurso convocado en diciembre del año anterior, de cuyo jurado debía formar parte. Pero antes de entrar a detallar las circunstancias en que se produjo esta nueva estancia sevillana del arquitecto milanés, conviene mencionar otro evento, desarrollado también en esos últimos meses de 1975, que contribuyó a afianzar su presencia en Andalucía y que nuevamente estuvo propiciado por el Colegio de Arquitectos. Me estoy refiriendo a la exposición «Arquitectura y racionalismo. Aldo Rossi + 21 arquitectos españoles» (Fig. 02), organizada por Salvador Tarragó con materiales provenientes de una muestra anterior, que había tenido lugar en Berlín ${ }^{27}$.

A los proyectos expuestos en la capital germana, se añadieron otros de jóvenes arquitectos espańoles que se podían considerar afines a los planteamientos rossianos, sintetizados con el término racionalismo que figuraba en el título de la exposición. La muestra se pudo visitar en Barcelona durante el mes de mayo de 1975 , pero aquella no fue más que la primera escala de un recorrido más largo, ya que había sido concebida con carácter itinerante ${ }^{28}$. No es extraño, pues, que Tarragó la ofreciera a aquellos Colegios de Arquitectos con los que tenía mayor contacto, comenzando por el de Andalucía occidental. Acordó con el CEYS que, en el último trimestre del año, la muestra recorriera las demarcaciones del Colegio, aunque finalmente sólo estuvo en tres de ellas: Cádiz, Córdoba 
y Huelva; si en un principio hubo la intención de que se exhibiera también en Sevilla, este propósito no llegó a concretarse ${ }^{29}$.

Vayamos ahora con el concurso que trajo de nuevo a Rossi a la capital andaluza. La junta general del Colegio de Arquitectos de Andalucía Occidental y Badajoz había acordado, en mayo de 1975, la construcción en Sevilla de un edificio de nueva planta al que habrían de trasladarse las oficinas de los servicios colegiales. Para dar cumplimiento a ese acuerdo, en los primeros días de diciembre la junta de gobierno se reunió con el fin de aprobar todo lo relativo al concurso de la nueva sede del Colegio. Las bases se hicieron públicas una semana después; entre otras cosas, determinaban los plazos dentro de los cuales el jurado habría de reunirse y emitir su fallo $^{30}$. Se presentaron trescientos proyectos de arquitectos provenientes de toda la geografía nacional (Fig. 03).

El jurado, compuesto por Julio Tirado de Serrano, José Antonio Coderch de Sentmenat, Aldo Rossi, Rafael Moneo Vallés, Luis Peña Ganchegui, José María García de Paredes Barreda y Roberto de Juan Valiente -Álvaro Siza Vieira, inicialmente designado, no pudo acudir-, se reunió entre el 24 y el 28 de abril, fecha en que está firmada el acta que recoge los proyectos premiados. Más allá de las discusiones que se produjeron en el seno del mismo en torno a los finalistas, resultan de interés las consideraciones que se dejaron consignadas por escrito sobre el tema del concurso, por cuanto ponen de manifiesto en qué medida las ideas rossianas estuvieron presentes en los debates del jurado. Se buscaba «un proyecto que plantease una alternativa arquitectónica ligada a los valores urbanos, a la forma de la ciudad, a todos aquellos elementos técnicos, constructivos y formales propios de Sevilla ${ }^{\otimes}$ : éste era el planteamiento con que el Colegio de Arquitectos había pensado el concurso, pero también el de Rossi en su proyecto para el Corral del Conde.

Rossi había viajado en avión desde Milán a Sevilla el 23 de abril, víspera de la constitución del jurado, y se alojó en un hotel con los restantes miembros del mismo $^{32}$. Su participación en ese concurso como miembro del jurado posibilitó que le conocieran personalmente algunos arquitectos espańoles de una generación anterior a la suya, que quizá no le apreciaban especialmente ${ }^{33}$; en particular, Coderch manifestó que aquellos días habían cambiado por completo su percepción del arquitecto italiano. Por lo demás, este viaje permitió a Rossi volver a la Feria, ir a los toros y retomar el contacto con los amigos sevillanos, con quienes ya compartía intereses y proyectos; pronto surgirían nuevas iniciativas, algunas de las cuales propiciarían que volviera a Sevilla en los años sucesivos.

Entre ellas, la más ambiciosa fueron sin duda los SIAC, iniciados precisamente ese ańo. El I Seminario Internacional de Arquitectura en Compostela estuvo organizado desde el recién creado Colexio Oficial de Arquitectos de Galicia, donde Salvador Tarragó había comenzado a encargarse del archivo histórico, y su tema fue "Proyecto y ciudad histórica». Se desarrolló en Santiago del 27 de septiembre al 9 de octubre y la dirección corrió a cargo de Rossi, que llamó como conferenciantes a un selecto grupo de arquitectos de reconocido prestigio ${ }^{34}$. Entre los participantes hubo una nutrida representación sevillana: Lino Álvarez Reguillo, Francisco Barrionuevo Ferrer, Gonzalo Díaz Recasens, Enrique de Haro Ruiz, Jaime Montaner Roselló, José Luis Palomino Romera, Aurelio del Pozo Serrano, Juan Ruesga y Fernando Villanueva; los dos últimos hablaron de la evolución urbana de la capital andaluza en una de las sesiones plenarias (Fig. 04). Además, Andalucía estuvo muy presente en la conferencia inaugural de Rossi, que utilizó en su exposición el ejemplo de la Feria y se refirió a Sevilla, Granada y Córdoba como «tres ciudades profundamente inmersas en mi fantasía, en mi memoria, en mis proyectos» ${ }^{35}$.

\section{7: LA CASA SEVILLANA}

En el imaginario de Rossi, los corrales de vecinos no tardaron en convertirse en el emblema de la arquitectura residencial de la ciudad. Así, en el texto introductorio para el proyectado libro sevillano, habla de como «las relaciones analógicas, las asociaciones entre las cosas y las situaciones, no han hecho sino aumentar en mis sucesivas estancias en Andalucía»; y añade: «Siempre me ha gustado la tipología del corral y la he representado muchas veces». Los corrales sevillanos le evocaban sin duda las galerías de las casas del viejo Milán, en cuyos patios entraba cuando era niño «con curiosidad y temor ${ }^{36}$; también por eso debió sentirse fascinado por la posibilidad de hacer un proyecto para el Corral del Conde.

Coincidiendo con esos primeros viajes de Rossi a Sevilla, un equipo de profesores de la Escuela de Arquitectura se encontraba estudiando la arquitectura doméstica del centro histórico de la ciudad. Durante los cursos 1973/74 y 1974/75 habían llevado a cabo, con sus alumnos de Elementos de Composición, una serie de levantamientos de casas-patio y corrales de vecinos. 

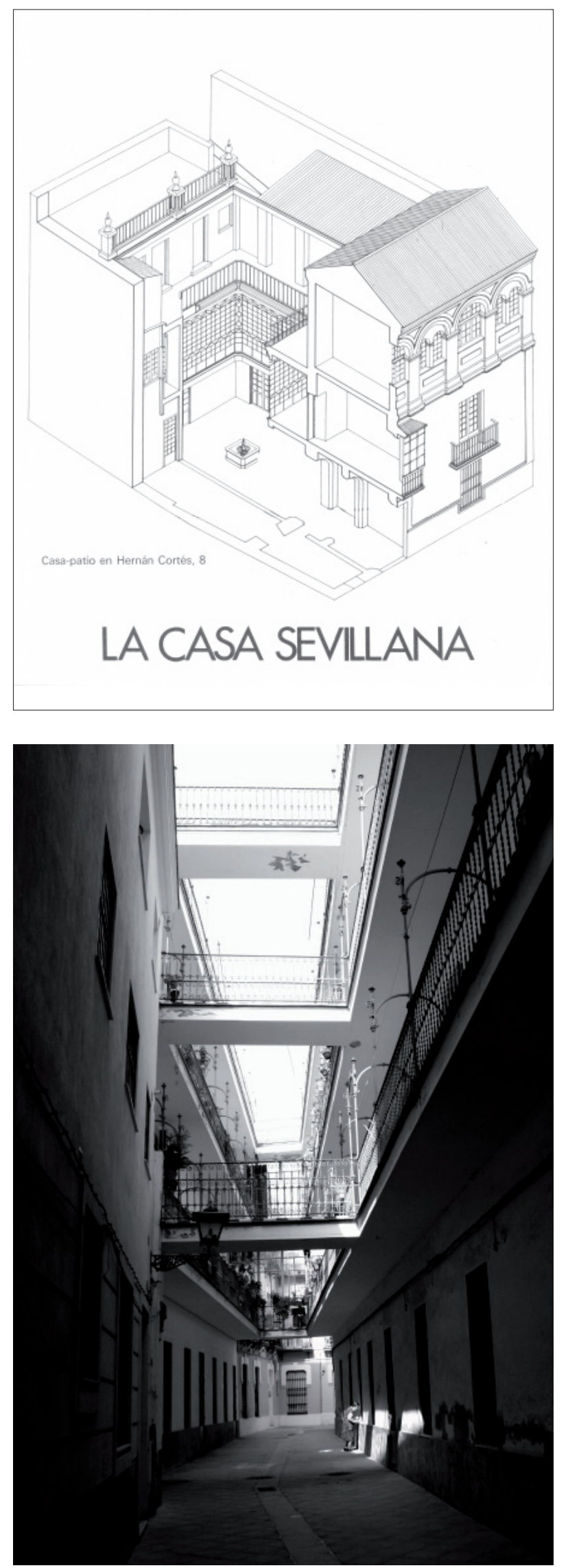

5 Folleto de la exposición "La casa sevillana", Sevilla (1977)

6 Manuel Martínez Mas y Ramón Balbuena, Pasaje de Valvanera, Sevilla (1915-25)
Ese trabajo se vio interrumpido por el apartamiento de la docencia de muchos de ellos en septiembre de $1975^{37}$. Fue entonces cuando Antonio Barrionuevo y Paco Torres crearon el seminario «Arquitectura y ciudad» como un instrumento que les permitiera continuar el trabajo iniciado y, con algunos alumnos que les facilitó el catedrático de la asignatura, siguieron dibujando y analizando aquellas casas. Esos levantamientos, junto con las fotografías que realizaron tres fotógrafos locales, sirvieron para montar la exposición «La casa sevillana» (Fig. 05) ${ }^{38}$.

Coincidiendo con la exposición, sus organizadores programaron un ciclo de conferencias dedicado a desarrollar algunos aspectos teóricos, históricos y metodológicos que facilitaran la comprensión del alcance de la investigación desarrollada. Las conferencias fueron tres: la primera corrió a cargo de Aldo Rossi, quien habló de "La arquitectura del casco antiguo de Sevilla»; la segunda la dio Víctor Pérez Escolano sobre el tema «Entre el rigor y la retórica. Casa y ciudad en la Sevilla moderna»; en la tercera, bajo el título «Nuevas consideraciones sobre la casa sevillana», Barrionuevo y Torres explicaron los presupuestos que habían guiado su trabajo. La intervención de Rossi tuvo lugar en el salón de actos de la Escuela de Arquitectura, mientras que las otras dos conferencias se impartieron en el salón de actos de la Caja de Ahorros que había financiado la exposición.

Rossi había traído unas notas con lo que pensaba decir en su charla, que completó, durante los días que pasó en Sevilla, con los materiales que le facilitaron Barrionuevo y Torres: una selección de imágenes de planos y fotografías de los edificios que se mostraban en la exposición y, sobre todo, un estudio sobre la casa sevillana publicado en 1928 por Joaquín Hazañas y La Rúa, del que Rossi extrajo un par de citas. Con todo ello, el arquitecto milanés completó el texto del que se sirvió para su exposición, en la que desarrolló su personal discurso sobre el proyecto y la ciudad histórica a partir de algunos ejemplos sevillanos: el hospital de la Caridad, la casa de los Bucarelli, algunos corrales —entre ellos, claro está, el del Conde-y el pasaje de Valvanera (Fig. 06), al que dedicó unas emocionadas palabras, probablemente las más citadas de esa conferencia ${ }^{39}$.

Barrionuevo y Torres tenían la idea de publicar un libro que recogiera el abundante material de que disponían; habían pensado titularlo, parafraseando a Joaquín Hazañas, «Nuevas consideraciones sobre la casa sevillana». Solicitaron diversas ayudas para su edición, 

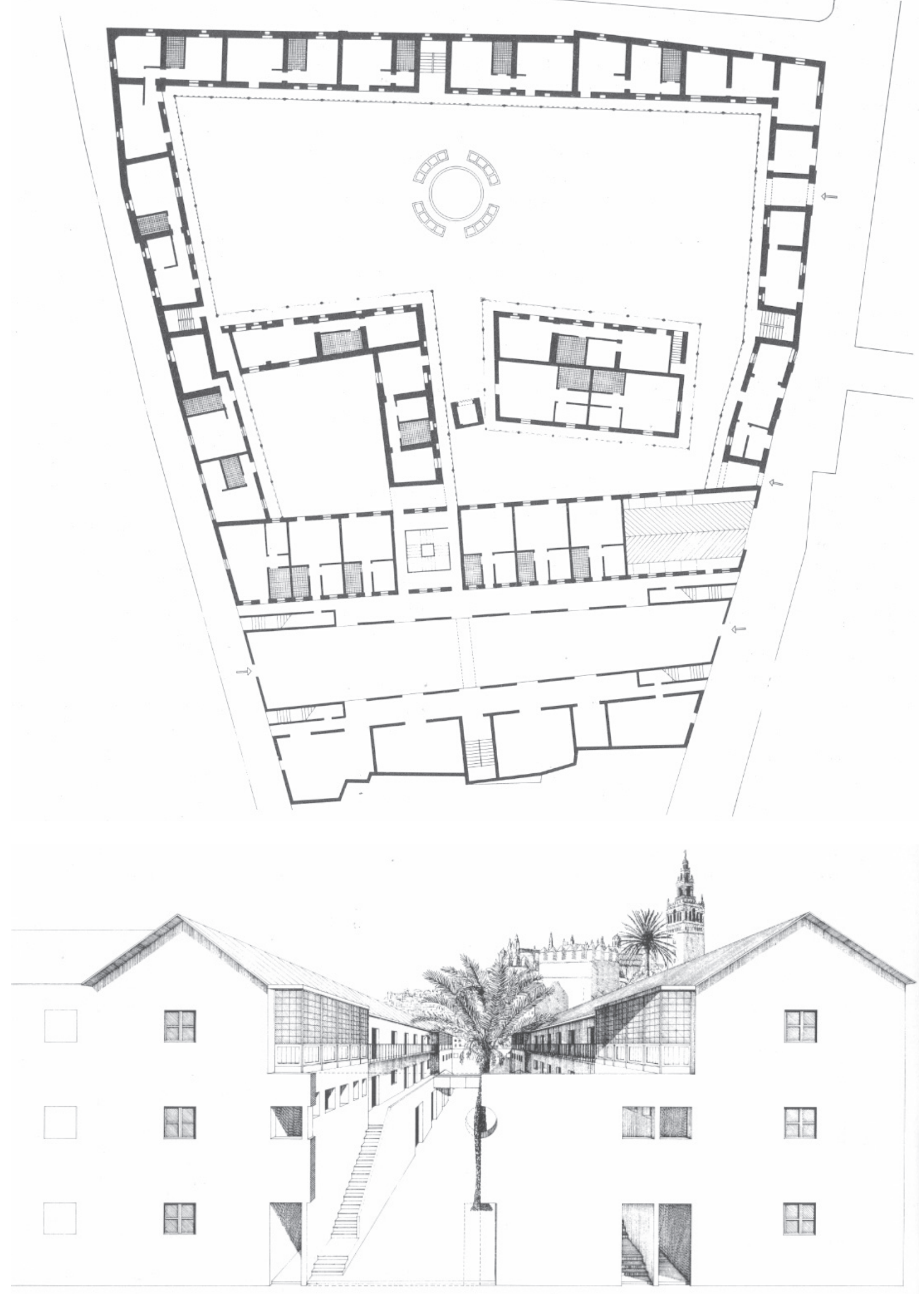

7-8 Aldo Rossi con Gianni Braghieri y Arduino Cantàfora, Corral del Conde, Sevilla (1977); planta del proyecto y perspectiva del pasaje 8 | Boletín Académico 3 (2013) 
pero no consiguieron la financiación que necesitaban, por lo que recurrieron a los amigos catalanes del Grupo 2C y les plantearon la posibilidad de hacer un número monográfico de su revista dedicado a la casa sevillana ${ }^{40}$. Ese número se publicó en 1978 y en el editorial del mismo se lee que «a los principales responsables de este estudio sobre la casa sevillana, Antonio Barrionuevo y Francisco Torres, nos unen vínculos estrechos de carácter personal y profesional. En varias ocasiones han estado presentes directa o indirectamente en la confección de esta revista. Así pues, para nosotros, el resultado de su trabajo constituye una comprobación de los planteamientos que hemos venido sosteniendo ${ }^{41}$.

De 1977 es también el proyecto definitivo para el Corral del Conde ${ }^{42}$. La visita que el arquitecto milanés realizó al corral en 1975 le hizo concebir un proyecto que, renunciando a la materialidad física del edificio existente, lo reconducía al esquema abstracto de su forma tipológica: a su juicio, lo que estaba en juego era «mucho más que unos arcos o unas piezas de hierro forjado, que podemos perder tranquilamente» ${ }^{43}$. Así, inicialmente, Rossi planteó la sustitución del edificio histórico por otro que mantenía su tipología ${ }^{44}$; sin embargo, tras una conversación con el arquitecto Rafael Manzano Martos, se decidió a restaurarlo (Fig. 07). Ayudándose de los materiales que recibió desde Sevilla ${ }^{45}$, tanteó diversas soluciones hasta llegar a la propuesta final, que incorporaba un pasaje situado sobre las parcelas colindantes, concebido como una especie de crecimiento espontáneo del corral (Fig. 08). Tampoco esta solución superaría la fase de anteproyecto: por diversas razones, el proyecto nunca llegó a realizarse ${ }^{46}$.

\section{8: LA CELEBRACIÓN DE UN SEMINARIO}

En enero de 1978 Rossi estaba de nuevo en Sevilla. Aunque hasta la fecha no he conseguido establecer con certeza el motivo de este viaje, todo parece indicar que debió tratarse de algo relacionado con el proyecto del Corral del Conde: fue tal vez entonces cuando el asunto naufragó definitivamente. En cualquier caso, lo cierto es que, aprovechando esa estancia se tuvo, en el estudio de Barrionuevo y Torres, una reunión para tratar de lo relativo a la celebración del II SIAC. La idea inicial era que los seminarios, organizados por el Colexio Oficial de Arquitectos de Galicia, tuvieran lugar en Santiago con una periodicidad anual; sin embargo, pronto se vio la conveniencia de crear una asociación independiente del Colegio, que permitiera tener una estructura organizativa autónoma y facilitara la obtención de fondos para atender los gastos derivados del desarrollo de los SIAC ${ }^{47}$.
El II SIAC estaba programado para la segunda quincena de septiembre de 1977, pero las dificultades económicas obligaron a retrasar su celebración ${ }^{48}$. A mediados de enero se reunieron con Rossi en Sevilla representantes de diversos grupos regionales para debatir lo relativo a la organización de la segunda edición del seminario (Fig. 09). Allí se decidió, entre otras cosas, que los SIAC tuvieran un carácter rotatorio, desvinculándose la APAC del colegio gallego y la sede del seminario de la ciudad de Santiago. Una primera consecuencia de este cambio de orientación fue que el peso de la dirección de los seminarios dejó de recaer personalmente en Rossi y pasó al grupo regional encargado de su organización. Las ponencias y comunicaciones debían correr a cargo de los propios participantes; se esperaba que esto contribuyera a una mejor articulación de los contenidos de cada seminario, construidos en torno a un tema central que convendría definir al término de la edición anterior, con el fin de que los grupos regionales pudieran trabajar a partir de entonces en esa dirección. Estas y otras cuestiones relacionadas con el ajuste del modelo hicieron que el II SIAC fuera definido como «un seminario de transición» ${ }^{49}$.

En el mes de marzo dieron comienzo, en la sede sevillana del Colegio de Arquitectos, las sesiones preparatorias del seminario. Pronto se vio que el margen de tiempo para la organización del seminario era escaso y se procedió a posponer su celebración hasta después del verano. Se decidió también mantener como tema genérico el del I SIAC, "Proyecto y ciudad histórica», estableciendo como tema específico para la segunda edición «Sevilla: transformaciones e intervenciones en una ciudad mediterránea». Los contenidos se distribuyeron en torno a cuatro tipos de actividades: ponencias directamente relacionadas con el tema del II SIAC, conferencias sobre cuestiones de interés general, trabajos de carácter propositivo y asambleas de la APAC para estudiar la modificación de sus estatutos. Con carácter general, las mańanas se dedicarían al trabajo de los grupos y las tardes a la presentación de conferencias y comunicaciones.

La organización no estuvo exenta de tensiones, relacionadas con el lugar de la sede del seminario y con la orientación de los trabajos. Hubo no pocos recelos respecto al centralismo sevillano por parte de los restantes arquitectos andaluces y una notable resistencia de algunos sectores a que el seminario pudiera servir para el afianzamiento de una orientación considerada formalista, en detrimento de otras cuestiones de carácter más propiamente político. Repasando los títu- 
los de las intervenciones recogidas en el programa, se pueden apreciar, junto a las ponencias de miembros destacados de la Tendenza, otras de arquitectos locales que evidencian estas diferencias; así, José Ramón Sierra Delgado, en su elogio de la destrucción de la ciudad, ironizaba sobre un supuesto combate entre la casa sevillana y las casas de Sevilla, o los onubenses José Ramón Moreno García y Tomás Curbelo Ranero se pronunciaban, más abiertamente, sobre la visión que desde la periferia se tenía de los arquitectos sevillanos ${ }^{50}$.

Finalmente, el II SIAC tuvo lugar entre el 26 de septiembre y el 6 de octubre (Fig. 10), y las diversas sesiones se desarrollaron en aulas de la Facultad de Geografía e Historia de la universidad hispalense. Rossi llegó a Sevilla en los días finales del seminario, tal vez porque su conferencia era la última programada. Habló de la estructura del territorio véneto, un tema que se correspondía con el que estaba desarrollando ese curso con sus alumnos de la Escuela de Arquitectura de Venecia $^{51}$. Si en su conferencia del año anterior había establecido un cierto paralelismo entre Milán y Sevilla, acudiendo a las semejanzas entre los corrales y las casas di ringhiera, en esta ocasión insistió en las similitudes entre Venecia y Sevilla: ambas habían crecido por partes, por lo que sólo analizando las collaciones sevillanas y los sestieri venecianos se podía llegar a comprender la estructura de sus respectivos centros históricos.

Rossi dibujaba así un triángulo analógico que vinculaba estrechamente estas tres ciudades, tan profundamente ligadas a su vida y a su arquitectura; estaba creando una geografía personal, hecha de analogías y objetos de afecto, que le permitiría en adelante recurrir a esos lugares de memoria para anclar allí sus proyectos. Por eso pudo decir con verdad: «En realidad no me siento extranjero en Sevilla; al contrario, el estudio de Sevilla ha sido muy importante en mi arquitectura y en mi teoría ${ }^{52}$; por eso mismo, la experiencia sevillana del maestro milanés no es un simple episodio, más o menos relevante, de la historia local de esta ciudad, sino una clave imprescindible para aproximarse al significado de su obra.

\section{A MODO DE CONCLUSIONES}

Hasta la fecha, a pesar de las numerosas referencias a Sevilla que se encuentran en los textos de Rossi, no se ha llevado a cabo un estudio sistemático de lo que supusieron para su arquitectura aquellas primeras estancias del maestro lombardo en la capital andaluza. Ese estudio desborda por completo los objetivos del pre- sente artículo, pero cabe avanzar a modo de conclusión algunas pinceladas respecto a la honda presencia de lo sevillano en los proyectos rossianos contemporáneos a los viajes aquí examinados. Me referiré únicamente a tres proyectos que considero especialmente significativos a este respecto.

En 1976, el arquitecto milanés fue seleccionado para el concurso restringido convocado para realizar una residencia de estudiantes en la ciudad italiana de Chieti. Como él mismo dejó anotado en sus cuadernos azules, la estructura del proyecto presentado por Rossi, junto con Gianni Braghieri y Arduino Cantàfora, era «exactamente igual a la de la Feria; las casas de los estudiantes son las casetas ${ }^{53}$. La referencia a las cabinas de las playas de la isla de Elba, también mencionada a menudo por su autor, no debe ocultar que el modelo urbano que subyace en este proyecto es la Feria sevillana, como una verdadera ciudad análoga, capaz de integrar las múltiples relaciones que las cosas evocaban en la desbordante imaginación del arquitecto milanés. Por si no estuviera claro, el albero del pavimento de la maqueta o las palmeras del dibujo titulado rousselianamente «Impressions d'Afrique» vienen a subrayar con mucha precisión ese ambiente sevillano de todo el proyecto.

El denominado Teatrino cientifico es un proyecto de 1978, realizado esta vez con Braghieri y Roberto Freno. Se trata una especie de pequeño teatro de la memoria en el que se asiste a la representación de las arquitecturas de Rossi. En su fachada encontramos una nueva referencia sevillana: el reloj detenido a las 5 de la tarde, la hora de la muerte del torero invocada por García Lorca en su llanto por Ignacio Sánchez Mejías: «A las cinco de la tarde. / ¡Ay qué terribles cinco de la tarde! $/$ ¡Eran las cinco en todos los relojes!», escribe el poeta granadino ${ }^{54}$. Una vinculación extraordinariamente rica de contenido, no sólo porque llega a la esencia misma de la concepción rossiana de la arquitectura de la ciudad como permanente proceso de construcción y destrucción de sus edificios, sino porque tanto de Rossi como de Sánchez Mejías se ha dicho que buscaron la muerte y tuvieron la que eligieron. Es difícil, por lo demás, imaginar una expresión más sintética de la incisiva fórmula compositiva rossiana — «la simple adición de lógica y biografía ${ }^{55}$ - que la representada por este singular proyecto.

El más célebre y afortunado de los proyectos rossianos, el Teatro del Mundo, fue construido para la Bienal de Venecia e inaugurado en noviembre de 1979. Aun cuando la crítica no haya sabido reconocerlo, ese 
proyecto contiene una velada alusión a los corrales de comedias y, por tanto, encierra una secreta relación con el Corral del Conde. He aquí lo que señaló Rossi en una de sus conferencias sevillanas: «El Corral del Conde es uno de los edificios que siempre me han impresionado más; tiene para mí el mismo valor que la mezquita de Córdoba o que un anfiteatro. Y, ciertamente, es un teatro; como dice Hazañas: "Se llamó corrales a los antiguos teatros, instalados todos ellos en patios. Como el teatro llamado del Coliseo, que dio nombre a su calle, no fue sino un gran patio habilitado para representaciones dramáticas" " ${ }^{56}$. Por eso, las galerías situadas sobre el graderío - en realidad, dos escaleras enfrentadas - daban al espacio interior de este Teatro un indudable aire sevillano.

Sirvan estos breves comentarios para poner de manifiesto en qué medida pudieron servir a Rossi sus viajes a Sevilla para dar forma a sus proyectos.

Pero esto no fue todo: la otra cara de la moneda corresponde al fuerte impacto que tuvo la presencia de Rossi entre nosotros. En aquellos años, sus frecuentes viajes a España y la publicación en castellano de su libro convirtieron al arquitecto milanés en un autor casi iniciático. La brillantez de sus planteamientos sirvió para aglutinar en torno a él -y al movimiento por él impulsado, la Tendenza - a un inquieto grupo de jóvenes arquitectos, interesado en renovar la arquitectura y en recuperar el papel social del arquitecto. Los SIAC fueron el cauce a través del cual se intentó canalizar esa relación, pero pronto el interés intelectual por las cuestiones suscitadas por el pensamiento rossiano se transformaría en imitación formal de su particular lenguaje arquitectónico.

No todos, sin embargo, siguieron ese camino. Frente a quienes con un punto de vista bastante superficial acabaron trivializando su legado, hubo otros arquitectos —en cuya arquitectura no hay el menor atisbo de rossismo - que supieron aprender la lección que su obra encierra y procuraron desarrollarla en sus propias obras con mayor o menor fortuna, pero siempre con inteligencia; no en vano algunos de ellos le habían escuchado decir en la clausura del Seminario de Compostela, cuando estaba comenzando a aparecer una especie de academia morfo-tipológica, que «el academicismo indigno y malo de muchas arquitecturas es ante todo estúpido» ${ }^{57}$. Las obras de algunos de aquellos arquitectos, expuestas en la mencionada exposición «Aldo Rossi +21 arquitectos españoles», constituyen un temprano ejemplo de lo
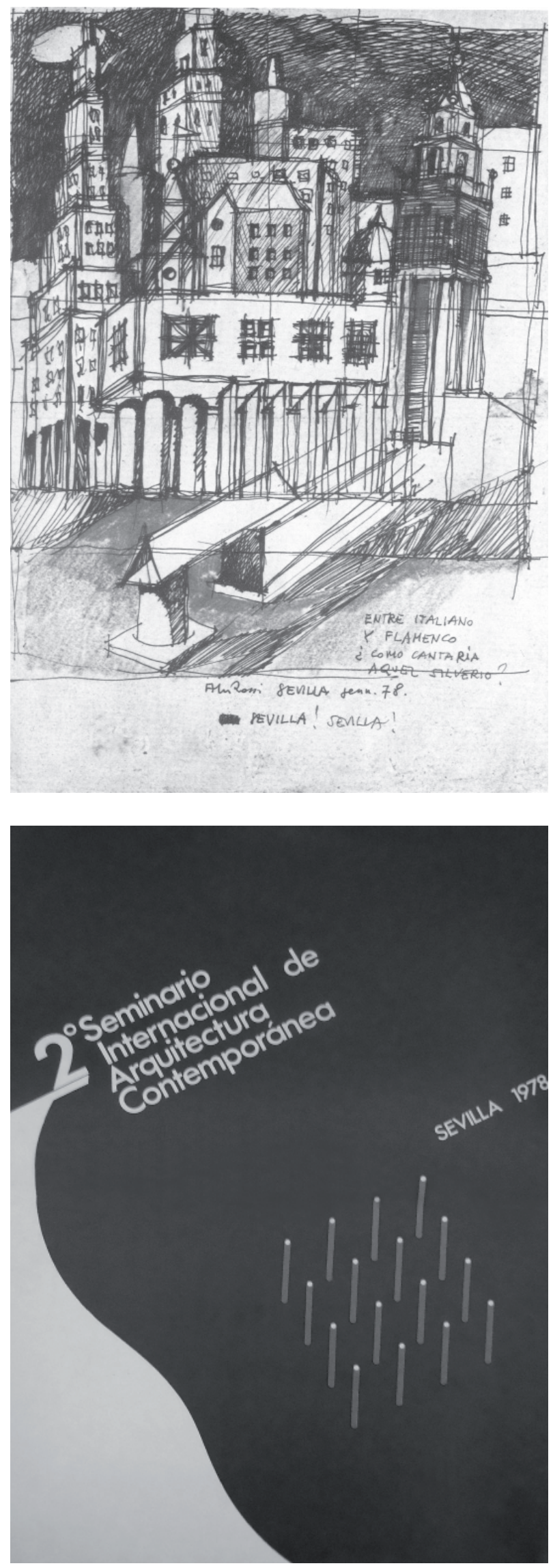

9 Aldo Rossi, "Sevilla, Sevilla" (1978); dibujo 10 Cartel anunciador del II SIAC, Sevilla (1978) 
que era un modo de situarse frente al magisterio rossiano ajeno por completo a la repetición banal de unos clichés formales.

En esta línea se movieron también los jóvenes arquitectos sevillanos que trataron más de cerca al maestro lombardo durante la segunda mitad de la década de 1970. Ciertamente, en aquellos ańos «tipologia edilizia y morfologia urbana son conceptos, que para una ciudad histórica como Sevilla, adquieren valor de conjuro" ${ }^{58}$, por cuanto el discurso teórico del arquitecto milanés se convirtió en un punto de referencia ineludible para el trabajo de muchos de los profesionales vinculados al CEYS: "Los arquitectos de mi generación, y sobre todo los andaluces — ha recordado Vázquez Consuegra—, se reconocieron especialmente en la gran lección de Aldo Rossi. [...] La figura de Rossi tuvo una gran influencia en la arquitectura andaluza». Y resumía esa lección diciendo que consistió en «la enseñanza de entender la ciudad como expresión formal de la historia y, por tanto, como referencia fundamen- tal para el proyecto arquitectónico. Esta contribución teórica condujo al deseo de construir una arquitectura más adecuada a nuestras ciudades, profundamente ligada a la realidad del lugar ${ }^{59}$.

Bastaría con revisitar los proyectos de arquitectos como Fernando Villanueva, Gonzalo Díaz Recasens, Antonio Barrionuevo, Francisco Torres o el propio Vázquez Consuegra, para comprobar que su italofilia era más conceptual que formal; los guiños lingüísticos presentes en aquellas primeras obras eran claramente deudores de un espectro más amplio de arquitectos entonces a la moda: desde Kahn a Venturi o los Five ${ }^{60}$. El influjo de Rossi se deja sentir, en cambio, de manera mucho más neta en otro tipo de trabajos, tales como la Guía de arquitectura de Sevilla de Vázquez Consuegra, publicada años más tarde pero iniciada en $1974^{61}$, o el reformado del Plan Especial del Casco Histórico de Sevilla, aprobado en 1980 y redactado por un equipo del que formaban parte Fernando Villanueva y Juan Ruesga.

Notas

1. Así, por ejemplo, a propósito del diverso papel que habían desempeñado en su formación la cultura norteamericana y la latinoamericana, Rossi llega a afirmar que se consideraba, "con orgullo y presunción, un hispanista» (Aldo Rossi, Autobiografía científica (Barcelona: Gustavo Gili, 1984), 90).

2. Carta de Rossi a Tarragó, 6 de septiembre de 1970 (Archivo Nacional de Cataluńa, San Cugat del Vallés (Barcelona): fondo 628, caja 8, dossier 28). Para una primera valoración del papel que la arquitectura espańola juega en la obra del arquitecto milanés, cf. Grupo 2C, «Aldo Rossi o la construcción dialéctica de la arquitectura», 2C Construcción de la Ciudad 2 (1975): 2-7.

3. A lo largo de esa década el libro tuvo cuatro ediciones (1971, 1976, 1977, 1979).

4. La revista, activa hasta 1985, dedicaría tres números monográficos a la obra de Rossi: 2 y 5 (1975) y 14 (1979), además de la entrevista recogida en el 0 (1972): 8-13.

5. Esas conferencias tuvieron lugar en Barcelona (enero de 1972), Santiago de Compostela (diciembre de 1973), San Sebastián (marzo de 1974), Sevilla, Granada y Córdoba (abril de 1975), y de nuevo en Santiago, con motivo del I SIAC (septiembre-octubre de 1976).

6. Sobre esas actividades de Tarragó, cf. Ignasi de Solà-Morales Rubió, «Memorias editoriales. Las traducciones españolas de Venturi y Rossi», Arquitectura Viva 18 (1991): 8; Marco Lecis, «Aldo Rossi e la Spagna. L'esperienza del Grupo 2C», en Annalisa Trentin (ed.), La lezione di Aldo Rossi (Bolonia: Bolonia University Press, 2008), 140-144.

7. Vincent Scully, «La ideología en la forma», en Alberto Ferlenga (ed.), Aldo Rossi: estudios críticos, (Barcelona: Serbal, 1992$), 136$.

8. Cf. Aldo Rossi, La arquitectura de la ciudad (Barcelona: Gustavo Gili, 1971), 89-90 y 216-218.

9. Carta de Rossi a Tarragó, 5 de julio de 1965. Archivo Nacional de Cataluña, San Cugat del Vallés (Barcelona): fondo 628, caja 8, dossier 28.

10. Francesco Dal Co, «Il teatro della vita. Introduzione ai quaderni azzurri di Aldo Rossi», en Aldo Rossi, I quaderni azzurri, $1968-1992$ (Milán: Electa \& Getty Research Institute, 1999), XI.

11. Rossi, Autobiografía cientifica, 28.

12. Loc. cit.

13. Estos dos proyectos, poco conocidos, fueron la rehabilitación de unas viviendas en el entorno del antiguo convento de Santa María de los Reyes y el Teatro de las Indias para la Expo'92. Lamentablemente, por diversas circunstancias, ninguno de los proyectos sevillanos de Rossi llegaría a ejecutarse.

14. La figura de Villanueva es clave para la relación sevillana con Rossi. Su figura está aún por estudiar; una primera aproximación a su trayectoria vital y profesional puede verse en Víctor Pérez Escolano, "Don Fernando Villanueva Sandino (1943-1992)", Cuadernos de la Alhambra 28 (1992): 9-14.

15. Entre otros casos que se podrían citar, Antonio Cruz Villalón, Antonio Ortiz García o Paco Torres eran arquitectos por la Escuela de Madrid, mientras que Antonio Barrionuevo había terminado en la Escuela de Barcelona.

16. Formaron parte del CEYS los arquitectos Lino Álvarez Reguillo (coordinador), Manuel Rodríguez Rivas (secretario técnico), Juan Ruesga (archivo histórico), José García-Tapial León(estudios urbanísticos), Manuel Alonso Gómez (biblioteca e información bibliográfica), Julio Tirado de Serrano (temas profesionales), Guillermo Vázquez Consuegra (formación y divulgación) y Antonio Barrionuevo (gabinete de prensa); la mayoría eran simultáneamente profesores de la Escuela de Arquitectura. Entre las numerosas cuestiones de actualidad que se abordaron en esos años desde el CEYS cabe recordar el debate sobre el futuro del Prado de San Sebastián (1974), la situación de los barrios del sector de Amate (1975), los derribos indiscriminados en el centro histórico (1975/76) o los problemas derivados de la corta de la Cartuja (1976).

17. Cf. «Declaración de Palma», Cuadernos de Arquitectura y Urbanismo 90 (1972): 3. Para contextualizar este asunto con los argumentos sevillanos de la época, cf. Luis Marín de Terán, «Sevilla: algunas notas sobre el casco antiguo», Arquitecturas Bis 3 (1974): 22-25; o Antonio Barrionuevo Ferrer, «Notas sobre la destrucción de la ciudad», 2C Construcción de la Ciudad 11 (1978): 48-49. 
18. Sobre la creación en Sevilla del archivo histórico del Colegio de Arquitectos, cf. Archivo FIDAS, Sevilla: CEYS, caja 8, dossier 08-2; un resumen de la reunión de Santiago, en el que queda constancia de la intervención de los arquitectos sevillanos, se conserva en ibid., dossier 08-4.

19. Declaraciones personales de Juan Ruesga Navarro al autor (marzo de 2012).

20. Declaraciones personales de Pilar Romero Valderas, viuda de Fernando Villanueva, al autor (febrero de 2012).

21. Rossi, I quaderni azzurri, \#18: Architettura, 8 gennaio 1975-12 giugno 1975.

22. La invitación, en nombre del CEYS, la había realizado Vázquez Consuegra, que fue quien se responsabilizó de su atención en esos días. Rossi pudo disfrutar de la Feria, ya que sus conferencias estaban programadas para la semana siguiente a su llegada a Sevilla. Declaraciones personales de Guillermo Vázquez Consuegra al autor (mayo de 2012).

23. En el archivo del Colegio de Arquitectos de Sevilla se conserva la nota del coordinador al gerente del CEYS, con fecha 2 de abril de 1975 , en la que se detalla el programa del viaje y el modo de repartir los gastos entre Sevilla, Granada y Córdoba (Archivo FIDAS, Sevilla: CEYS, caja 5). En el boletín mensual que editaba el CEYS, correspondiente a marzo de 1975, se informa que las conferencias de Rossi tendrían lugar los días 22 y 23 de abril.

24. El texto y la carta que lo acompańaba están en el archivo del Colegio de Arquitectos de Sevilla, junto a una fotocopia del contrato editorial para la publicación de sus Scritti scelti, que Rossi envió por si podía servir de modelo para la edición del proyectado libro sevillano (Archivo FIDAS, Sevilla: CEYS, caja 6); una memoria de las actividades realizadas y previstas por el departamento de formación y divulgación del CEYS, elaborada en octubre de 1975 , recoge la estructura de la publicación proyectada (ibid., caja 5).

25. Un breve resumen de los objetivos de Pro-Sevilla se puede ver en la nota introductoria, firmada por Ignacio Medina Fernández de Córdoba, que sirve como pórtico al libro de Alberto Corazón Climent (coord.), La vida en el barrio. Estudio sobre formas de vida y modelos urbanos en Sevilla y su casco histórico (Sevilla: Pro-Sevilla, 1979), 1-2.

26. Declaraciones personales de Gonzalo Díaz Recasens al autor (febrero de 2012). Sobre OTAISA en esos años, cf. Francisco J. Montero Fernández, "Conversaciones sobre OTAISA (I), de los sesenta a los setenta", en Varios autores, Arquitectura del racionalismo en Sevilla. Inicios y continuidades (Sevilla: COAS, 2003), 100-113.

27. En septiembre de 1974, Rossi había participado en el simposio «Das Pathos des Funktionalismus. Berliner Architektur 1920-1930», organizado por el Internationales Design Zentrum de Berlín; en paralelo se celebró la exposición «Aldo Rossi: Architektur des Rationalismus», que pudo visitarse en los locales del IDZ del 7 al 15 de septiembre.

28. Más datos sobre esa muestra en el epígrafe «Arquitectura y racionalismo, una exposición de los proyectos del grupo» de la revista $2 C$ Construcción de la Ciudad 8 (1977): 22-51, que incluye un artículo en el que se responde a las críticas recibidas, el texto escrito por Rossi para la ocasión y una selección de los proyectos de los arquitectos espańoles.

29. La carta del coordinador del CEYS a Tarragó, de fecha 4 de diciembre de 1975, comunicándole que se había realizado la transferencia bancaria con el importe de los derechos que convinieron por la cesión de la exposición, deja claro que ésta se había celebrado «en las ciudades de Cádiz, Córdoba y Huelva» (Archivo FIDAS, Sevilla: CEYS, caja 5).

30. La fecha límite para la entrega de los proyectos era el 19 de abril de 1976 y el jurado había de constituirse antes del día 25 , debiendo emitir su fallo no más tarde del 9 de mayo.

31. Varios autores, Concurso de anteproyectos para la nueva sede social del Colegio Oficial de Arquitectos de Andalucía occidental y Badajoz (Sevilla: COAAOB, 1976), 117.

32. Así se desprende de los documentos conservados en la carpeta «Materiale diverso Spagna», donde entre otras cosas se halla el billete de avión para ese viaje (Archivo MAXXI, Roma: AR-6.DID/036)

33. Conviene recordar a este propósito que en aquel momento los planteamientos de Rossi tenían todavía un marcado carácter de tendencia, es decir, aspiraban a intervenir en la batalla de las ideas desde una posición precisa, tanto desde el punto de vista político como disciplinar. Este planteamiento militante despertaba necesariamente adhesiones y enemistades, con la consiguiente aparición de fuego cruzado desde diversos frentes; una muestra de esta dimensión polémica del personaje se puede ver, por ejemplo, en la crónica del I SIAC realizada por Salvador Fraga Rivas y Julio Malo de Molina Martín-Montalvo, «Aldo Rossi: una alternativa progresista para la arquitectura», Triunfo 719 (1976): 49.

34. Intervinieron, entre otros, C. Aymonino, J. Stirling, O.M. Ungers, J.P. Kleihues o M. Gandelsonas.

35. Aldo Rossi, "Ciudad y proyecto», en Varios autores, Proyecto y ciudad histórica. I Seminario Internacional de Arquitectura en Compostela (Santiago de Compostela: COAG, 1977), 23 y 19.

36. Aldo Rossi, «Sevilla», texto inédito de 1976 (Archivo MAXXI, Roma: AR-6.DID/039), luego incluido en Rossi, Autobiografía científica, 28.

37. Cf. Juan Luis Trillo de Leyva, De memoria. Orígenes de la Escuela de Arquitectura de Sevilla (Sevilla: Universidad de Sevilla, 2010), $233-243$.

38. La exposición tuvo lugar entre el 5 y el 27 de mayo de 1977.

39. Esas palabras las dejó anotadas el propio Rossi en uno de sus 'cuadernos azules': "Como decía hablando de Valvanera, la casa sevillana la considero como el mejor de mis proyectos" (Rossi, I quaderni azzurri, \#21: Architettura. USA/Progetto Firenze/Venezia, 20 ottobre 1976-15 maggio 1977).

40. Declaraciones personales de Antonio Barrionuevo Ferrer al autor (febrero de 2012).

41. Grupo 2C, «Editorial», 2C Construcción de la Ciudad 11 (1978): 6. Hay que señalar que las dos únicas obras de arquitectos andaluces seleccionadas para la exposición "Aldo Rossi + 21 arquitectos espańoles" —y luego publicadas en $2 C$ - eran de Barrionuevo y Torres.

42. En la portada de $A B C$ Sevilla, 18 de marzo de 1977, se lee: «El Corral del Conde, a salvo. El famoso y popular Corral del Conde, que mandó construir para viviendas de sus criados el conde-duque de Olivares, será restaurado cuidadosamente y, junto con un solar contiguo, transformado en una espléndida residencia, que conserve todo su sabor y los magníficos detalles constructivos que lo hicieron célebre»; en el interior de ese número no se amplía la noticia. No lo he podido documentar, pero supongo que a esa fecha debe corresponder la presentación en Sevilla del proyecto de Rossi.

43. Aldo Rossi, «La arquitectura del casco antiguo de Sevilla», 4, conferencia impartida el 9 de mayo de 1977 dentro del ciclo "La casa sevillana» (Archivo MAXXI, Roma: AR-6.DID/024).

44. «La demolición del corral, de estructura de muros de carga y viguería de madera en mal estado, permite una reconstrucción en la que se reconsidera la dimensión de la crujía y se resuelven los forjados y la galería con perfiles metálicos» (Paco Torres, «Nota sobre un proyecto para Sevilla», 2C Construcción de la Ciudad 14 (1979): 23).

45. Una selección bibliográfica sobre los corrales y algunos levantamientos, realizados en su mayoría por alumnos de la Escuela de Arquitectura, se conservan en el archivo del MAXXI (AR-1.PRO/031). Le fueron remitidos por Fernando Villanueva poco después de la primera estancia sevillana de Rossi; en la carta que los acompańaba se puede leer: «Estoy preparando una nota sobre el desarrollo histórico del Corral del Conde, que te enviaré cuando la tenga terminada y si necesitas algún otro tipo de información estaré encantado de podértela facilitar» (carta de Villanueva a Rossi, 10 de mayo de 1975, conservada en el archivo de Fernando Villanueva Sandino).

46. Para la historia del proyecto, cf. Gonzalo Díaz Recasens y Fernando Villanueva, «El Corral del Conde o la Sevilla de Rossi», Jano Arquitectura 61 (1978): 55-56

47. La Asociación para el Progreso de la Arquitectura Contemporánea (APAC) fue fundada en junio de 1977 en Santiago de Compostela, aunque su constitución como entidad con personalidad jurídica no se produjo hasta unos meses más tarde. Toda la documentación manejada en relación con el II SIAC procede del archivo de Antonio Barrionuevo Ferrer. 
48. En relación con los temas que aquí nos ocupan, es interesante constatar que, según la documentación disponible sobre el II SIAC, en el programa inicial estaba prevista una conferencia de Rossi dedicada al proyecto para el Corral del Conde; se barajaba asimismo la posibilidad de contar con la exposición «La casa sevillana».

49. Informe sobre la reunión preparatoria del II SIAC, tenida en Sevilla, del 19 al 21 de enero de 1978; una copia de ese informe fue remitida a los socios de la APAC mediante carta circular de Tarragó, de fecha 1 de febrero (Archivo Barrionuevo, carpeta II SIAC).

50. A diferencia de lo sucedido con los otros dos SIAC españoles —el de Santiago y el de Barcelona—, no se llegó a editar ningún volumen que recogiera los trabajos desarrollados en el de Sevilla; aunque me consta que se hicieron diversos intentos para publicarlos, desconozco si se llegaron a recoger los materiales elaborados en esos días. Entre las reseñas del II SIAC, cf. Fernando Villanueva, "Crónica del II SIAC», Arquitectura 214 (1978): 6; Grupo 2C, «Balance y perspectiva del II SIAC, después de Sevilla», 2C Construcción de la Ciudad 12 (1978): 64-65; Carlos Almuiña Díaz, "De Compostela a Contemporánea, ou o que vai de Santiago'76 a Sevilla’78. Crónica do II SIAC», Obradoiro 2 (1978): 53-59.

51. Cf. Aldo Rossi et al., Le città venete. Architettura e territorio. Matteriali didattici e di ricerca del gruppo di lavoro diretto dal prof. Aldo Rossi, anno accadèmico 1977-78 (Venecia: IUAV, 1978).

52. Rossi, «La arquitectura del casco antiguo de Sevilla», 1.

53. Rossi, I quaderni azzurri, \#19: Architettura. 6 settembre 1975-luglio 1976.

54. Es como si, durante esos años al menos, Rossi se viera a sí mismo — Lorca de nuevo- «entre italiano y flamenco»; no en vano el maestro lombardo dejó anotados los primeros versos del «Retrato de Silverio Franconetti» en uno de sus dibujos sevillanos de 1978, aquí reproducido (Fig. 09).

55. Rossi, Autobiografía cientifica, 18.

56. Rossi, «La arquitectura del casco antiguo de Sevilla», 8; la cita recogida por Rossi está tomada del estudio de Joaquín Hazañas de la Rúa sobre la casa sevillana (1928)

57. Aldo Rossi, «Palabras en la clausura del I SIAC», en Proyecto y ciudad histórica, 293-294.

58. Víctor Pérez Escolano, «La escuela del toreo de salón: la joven arquitectura sevillana», Arquitectura 210 (1978): 9.

59. Tommaso Vecci (ed.), Trentotto domande a Guillermo Vázquez Consuegra (Nápoles: CLEAN, 2007), 17 y 20.

60. Cf. por ejemplo, la selección de proyectos de esa joven generación de arquitectos sevillanos recogida en Jano Arquitectura 56 (1978): $24-58$, y el comentario de Víctor Pérez Escolano que la precede («Los jóvenes arquitectos del rigor», idem., 20-23).

61. Guillermo Vázquez Consuegra, Guía de arquitectura de Sevilla (Sevilla: Junta de Andalucía, 1992).

\section{Procedencia de las ilustraciones}

Fig. 01, 04 y 10. Archivo Fernando Villanueva Sandino, Sevilla.

Fig. 02, 05 y 06. Archivo Antonio Barrionuevo Ferrer, Sevilla.

Fig. 03. Archivo Fundación FIDAS, Sevilla.

Fig. 07 y 08. Alberto Ferlenga, Aldo Rossi: architetture 1959-1987 (Milán: Electa, 1987) (C) Eredi Aldo Rossi, Courtesy Fondazione Aldo Rossi.

Fig. 09. Archivo del MAXXI, Roma @ Eredi Aldo Rossi, Courtesy Fondazione Aldo Rossi.

\section{Sobre el autor}

Victoriano Sainz Gutiérrez (Madrid, 1961) es arquitecto por la Universidad de Sevilla, donde también se doctoró con una tesis sobre Aldo Rossi. En la actualidad ejerce como profesor titular en la ETSA de Sevilla, de cuyo Departamento de Urbanística y Ordenación del Territorio es director. Ha publicado, entre otros, los siguientes libros: La cultura urbana de la posmodernidad. Aldo Rossi y su contexto (1999), El proyecto urbano en España. Génesis y desarrollo de un urbanismo de los arquitectos (2006) y Aldo Rossi: la ciudad, la arquitectura, el pensamiento (2011). 